\section{From Mirror to Mist: Cracking the Secret of Fracture Instabilities}

When cracks in a material propagate, bonds between atoms are broken, generating two new surfaces. Experiments have shown that cracks moving at low speeds create atomically flat, mirror-like surfaces, whereas cracks moving at higher speeds create increasingly rough fracture surfaces. Dynamical instability leads to increasing roughening transitioning from a mirror-like surface to a less reflective (mist) surface to a very rough irregularly faceted (hackle) surface. Using massively parallel large-scale atomistic simulations, Markus J. Buehler from the Massachusetts Institute of Technology (MIT) in Cambridge, Mass., and Huajian Gao from the Max Planck Institute for Metals Research in Stuttgart, Germany, (now at Brown University) have developed a new theoretical model to understand atomistic details of how cracks propagate in brittle materials, revealing the physics of dynamical fracture instabilities. As reported in the January 19 issue of Nature (p. 307; DOI: $10.1038 /$ nature 04408), their models indicate, in contrast to current theories, that it is critical to consider the properties of materials at large deformations, close to the crack tip, in order to understand how materials fracture. These findings have major implications for the understanding of fracture at different scales, ranging from the nanoscale to the larger scales of airplanes, buildings, or even earthquake dynamics, and predict that cracks can move at speeds faster than the velocity of sound, which has so far been considered an impenetrable barrier for crackpropagation speed.

The deformation and fracture of materials has fascinated scientists for decades, due to both their scientific relevance as well as their significance in engineering and to society. In the past, the classical physics of the continuum has been the basis for most theoretical and computational tools in engineering analyses of these phenomena, and theories relying on numerous phenomenological assumptions have been used. Scientists are now beginning to use atomistic simulation as a tool to study the behavior of materials under extreme conditions in order to gain insights about the fundamental mechanisms of deformation and failure at length and time scales unattainable by experimental measurements and which cannot be predicted using continuum theories.

This phenomenon of greater roughness for faster crack propagation is found in many different classes of brittle materials, including metals, polymers, and semiconductors, at a variety of scales. Until now, no sound understanding of the underlying physics or of the particular crack speed at which the instability occurs has been achieved. None of the existing theories explain

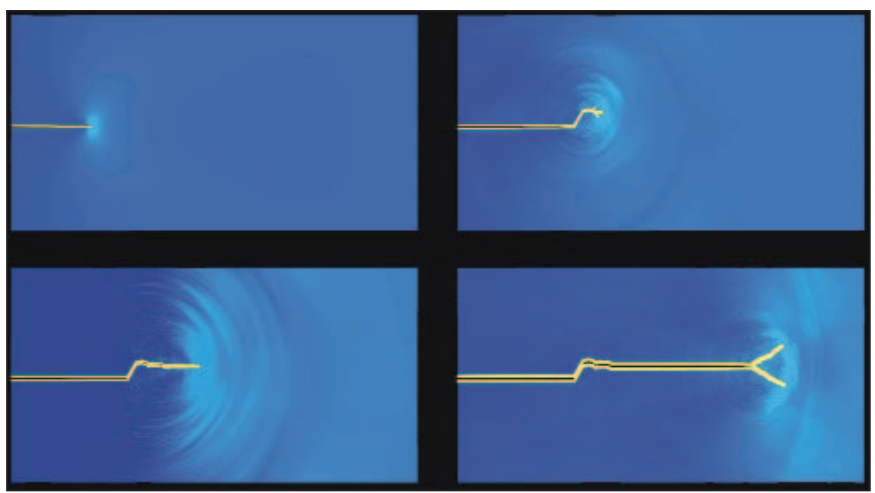

Figure 1. Atomistic simulation shows the dynamical sequence as fracture instability occurs. At a critical crack speed, straight crack motion becomes unstable and the crack starts to wiggle, creating increasingly rough surfaces. Courtesy of Markus J. Buehler, MIT.

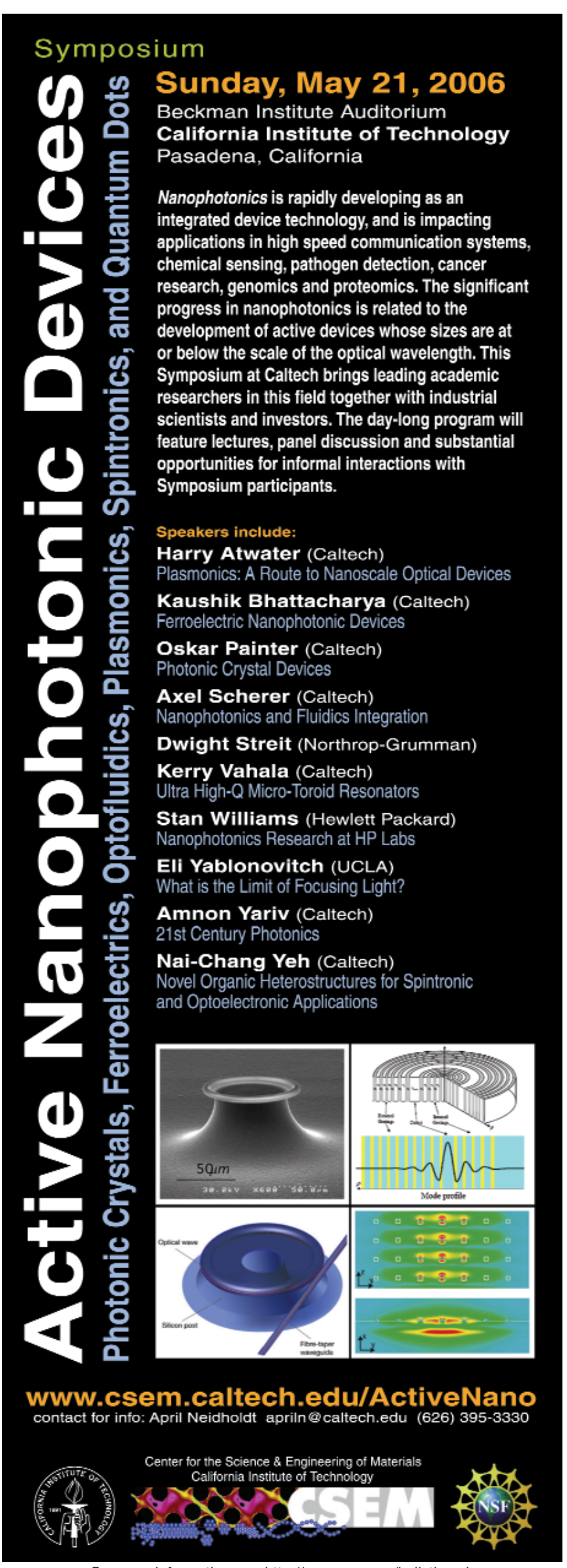

For more information, see http://www.mrs.org/bulletin_ads 
the wide range of experimental and computational results, and many models contradict each other.

By means of large-scale atomistic simulations, Buehler and Gao show that hyperelasticity, the elasticity at large strains, can play a governing role in dynamical crack tip instabilities in the fracture of brittle materials. They report a modified instability model that treats the dynamical fracture instability as a competition between different mechanisms controlled by local energy flow and local stress field or atomic forces near the crack tip. Their results suggest that the fracture instability not only appears in materials with defects; it is an intrinsic phenomenon of dynamical fracture. See Figure 1 (p. 167).

"Our new theory reduces to existing models in limiting cases, but allows for a unified treatment of the instability problem applicable to a much wider range of materials," said Buehler. "We have discovered that the key to understanding the discrepancies in the literature is to consider the material behavior close to the breaking of bonds, rather than the material properties at small strains."
Most existing theories of fracture assume a linear elastic stress-strain law by only considering small strain deformation. However, the relation between stress and strain in real solids is strongly nonlinear due to large deformation near a moving crack tip, a phenomenon referred to as hyperelasticity or nonlinear elasticity.

The scientists have made another surprising discovery. "We find that elastic stiffening materials behavior, as found in rubber-like materials, can dramatically change the instability dynamics of cracks," Buehler said. Rubber is soft at small deformations, and becomes harder as the stretch is increased. "In such elastically stiffening materials, stable intersonic crack motion is possible," he said. These results are in contrast to existing theories, in which the speed of elastic waves is considered the limiting speed of fracture, analogous to the speed of light.

"We discovered that the classical theories of instability dynamics are only valid in a small range of material behavior," said Buehler. "In most real materials, the softening or stiffening close to bond breaking leads to a fundamental change in the instability dynamics, because energy flow is reduced or enhanced due to change in local wave speed."

These results represent a breakthrough in understanding how cracks propagate in brittle materials, and the findings could have wide impact in many scientific and engineering disciplines, said the researchers.

\section{Scanning Ultrasound Holography Allows Nanoscale Subsurface Imaging}

The characterization of deeply buried or embedded structures and features with lateral resolution of under $100 \mathrm{~nm}$ is critical in a number of science and engineering arenas. Subsurface features can be imaged with techniques such as acoustic microscopy, but with limited spatial resolution. More recently, scanning probe techniques, such as ultrasonic force microscopy, have been used for nanomechanical mapping of elastic and viscoelastic properties of surfaces. However, these techniques still lack the sensitivity required for imaging buried structures. Now, Gajendra Shekhawat

\section{Announcement and Call for Papers}

\section{October 17-19, 2006, in San Diego, California Advanced Metallization Conference (AMC) 2006}

This conference is the 23rd in a series devoted to leading-edge research in the field of advanced metallization and 3-D integration for IC applications. Papers may be submitted on topics affecting state-of-the-art and future directions in interconnect systems, including:

Metallization, material science, and interfaces Advanced deposition techniques and kinetics Nucleation and adhesion studies Diffusion barrier performance

Interconnect thin film microcharacterization Electrical and mechanical properties Morphology evolution and stability

Advanced semiconductor device architecture Low-k dielectrics

Metal and dielectric thin film barriers High-k and ferroelectric capacitors

Inductors, capacitors, etc., in the wiring levels

Advanced wiring schemes (e.g., for embedded DRAM, system-on-a-chip, etc.) Vertical (3-D) interconnects Nano-interconnects (CNT)

Metal gate transistors design, fabrication, characterization, and reliability

RF interconnects

Interaction of packaging with on-chip interconnects
Process modeling

Advancements in CVD and PVD deposition

Electrochemical and electroless deposition

Chemical mechanical polishing

Novel deposition and planarization techniques

Multilevel process integration issues

System-on-a-chip

3-D system integration

Novel interconnect system concepts

Chip interconnect/packaging interface issues

MEMS metallization issues and solutions

Advanced patterning and etching processes

Damascene and dual-damascene techniques

Barrier/liner/fill technology

Integration of $\mathrm{Cu}$ and $\mathrm{Al}$ with low-k dielectrics

Reliability and performance data and simulations

Dual damascene process defects

Electromigration and stress migration

Dielectric reliability and diffusion studies

Adhesion, corrosion, and other stress testing

Reliability of active chips with Cu and/or low-k

Bonding and packaging issues for advanced interconnects
Abstracts are due June 2, 2006.

Send abstracts (two pages, 500 words, with supporting figures on second page) to Jenny Black Deer, UC Berkeley Extension, 1995 University Ave., Berkeley, CA 94720-7010; fax: (510) 642-6027; e-mail: amc@unex.berkeley.edu. Please note: all abstracts submitted electronically must be a pdf file (all fonts must be embedded in the file). Include the author's name, affiliation, mailing address, e-mail address, and phone and fax numbers on the abstract.

For an announcement brochure:

Call (510) 642-4151, fax (510) 642-6027, e-mail amc@unex.berkeley.edu, or write to: Continuing Education in Engineering, UC Berkeley Extension, 1995 University Ave., Berkeley, CA 94720-7010. Or visit www.unex.berkeley.edu/eng/metal
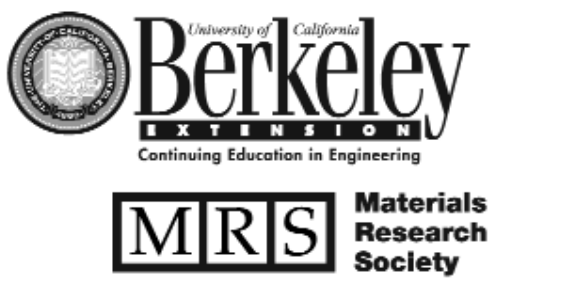

An MRs-affiliated meeting

For more information, see http://Www.mrs.org/bulletin_ads 